1986 and "Reduction of bistatic scattering matrix measurements for inversely symmetric radar targets," IEEE Trans. Antennas Propagat., vol. AP-31, no. 2, pp. 237-242, Mar. 1983.)

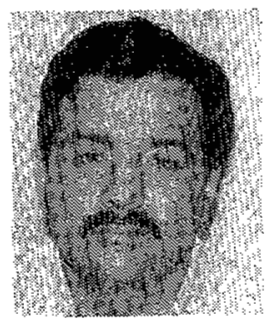

Alexander B. Kostinski was born in Kiev, USSR, on January 7, 1957. He received the B.Sc. degree in mathematics from the Hebrew University of Jerusalem in 1978 and the Ph.D. degree in theoretical physics (fluid dynamics) from the University of Illinois at Chicago in 1984.

He has held an appointment as a Student Research Associate at the Argonne National Laboratory while investigating the air flow patterns over a complex terrain. He is currently a Postdoctoral Research Associate at the Communications Laboratory of the Electrical Engineering and Computer Science Department at UIC, where he is working on various aspects of radar polarimetry, millimeter wave propaga- tion, and statistical optics and radiophysics. He has written papers on medical applications of fluid mechanics theory, on high Reynolds number theory of turbulence and on fundamentals of radar polarimetry. His interests also include crystal optics, in particular, propagation through liquid (cholesteric) crystals, atmospheric physics and propagation through ionospheric plasmas, and modern theory of chaotic phenomena.

Dr. Kostinski is a member of the American Physical Society.

Wolfgang-Martin Boerner (S'66-M'67-SM'75-F'84), for a photograph and biography please see page 2 of the January 1984 issue of this TRANSACTIONS.

\title{
Reduction of the Anomalous Resonances of Symmetric Log-Periodic Dipole Antennas
}

\author{
ZHONG-LIN GONG AND KEITH G. BALMAIN, SENIOR MEMBER, IEEE
}

\begin{abstract}
Computer analysis reveals that the anomalous behavior of symmetric log-periodic dipole antennas (LPDA) is mainly due to the radiation of the dipole elements in the anomalous radiating region which is excited by the transmission line resonance of the feed structure. By replacing the cylindrical dipole elements in the LPD antennas with wideangle triangular-outline dipoles with central wires, the computed anomalous back lobe peaks are reduced to negligible levels. LPD antennas were constructed using wide-angle triangular-outline dipoles with central wires, and they were found to exhibit satisfactory performance.
\end{abstract}

\section{INTRODUCTION}

$\mathrm{O}$ NE OF THE MAIN drawbacks of symmetric log-periodic dipole antennas (LPDA) is the presence of anomalous behavior, which is characterized by high back lobe levels over narrow frequency bands. The cause of this behavior is apparently the excitation of a resonator comprising the section of feed line between the reactive large-end termination (usually a short circuit) and the stop region which exists just behind the $\lambda / 2$ dipole due to heavy inductive loading

Manuscript received July 8, 1985; revised May 29, 1986. This work was supported by Natural Sciences and Engineering Research Council of Canada Grant A-4140.

Z. Gong was with the Department of Electrical Engineering, University of Toronto, Toronto, Canada. He is now with the Department of RadioElectronics, Peking University, Beijing, The People's Republic of China.

K. G. Balmain is with the Department of Electrical Engineering, University of Toronto, Toronto, Canada M5S IA4.

IEEE Log Number 8610040. there. Although several investigations have been carried out on this phenomenon and some remedies proposed, except for attaching a matched terminating resistor to the feeder at the large end of the antenna, the effective remedies were limited to choosing a sufficiently high scale factor $\tau$ or choosing the optimum feed line characteristic impedance $Z_{0} \sim 200 \mathrm{ohms}$ [1], [2]. This paper addresses the open question of how much $\tau$ and $Z_{0}$ both can be lowered while retaining a reactive feed line termination and maintaining the anomalous back lobes at a low level. The method chosen to achieve this is the alteration in shape of the dipole elements.

\section{Computer Analysis of Log-Pertodic Dipole Antennas}

\section{Structure and Parameters of Analyzed Models}

Two eight-element log-periodic dipole antennas were chosen as the models to be analyzed by computer. LPDA-1 has the following parameters: scale factor $\tau=0.90$, dipole spacing parameters $\sigma=0.069$, apex half-angle $\alpha=20^{\circ}$, and structure bandwidth $B_{s}=2.0$. The parameters of LPDA-2 are the following: $\tau=0.86, \sigma=0.087, \alpha=22^{\circ}$, and $B_{s}=$ 2.87. The two antennas were designed as illustrated in Fig. 1, with the longest dipole element a half-wavelength at $500 \mathrm{MHz}$ and the feed line terminated with a short circuit at a distance $s$ $=15 \mathrm{~cm}$ which is half the length of the longest dipole element. These moderately low values of $\tau$ (especially for LPDA-2) and the relatively long distance from the termination 


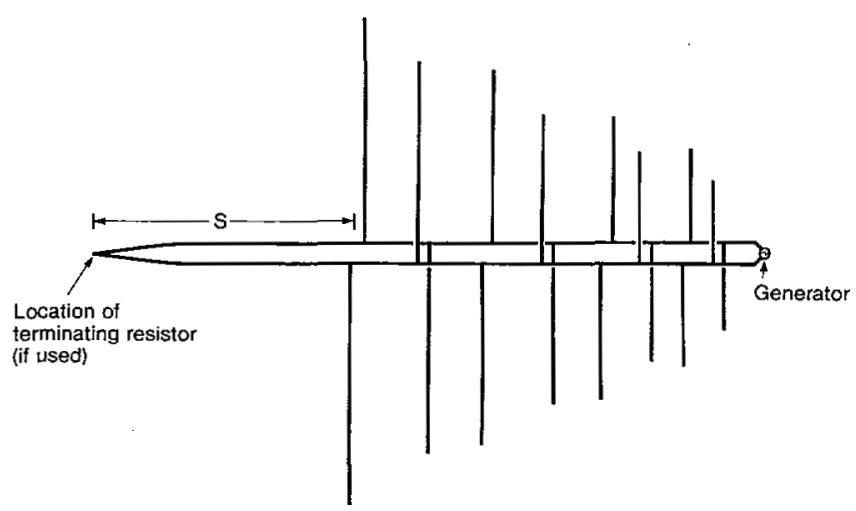

Fig. 1. Geometry used for LPDA computation.

to the longest element were deliberately chosen for making the anomalous behavior noticeable (see [1]). Richmond's computer program for thin-wire structures [3] was chosen to perform the computations, but in this program there exists the limitation of a unique wire diameter, so these antennas were designed such that all the dipole elements and feed line wires have the same diameter. In order to perform the computation, each antenna is divided into 66 segments, two segments for each monopole, one segment for each section of feed line between monopoles and eight segments for each feed line section from the termination to the longest dipole element.

Considering that the characteristic impedance $Z_{0}$ of the feed line has a significant effect on the anomalous behavior [2], the computer analysis was performed with the two antennas having low $Z_{0}$ (LPDA-1: $Z_{0}=106 \mathrm{ohms}$; LPDA-2: $Z_{0}=104$ ohms). Under the limitation of thin-wire structures, the respective values of the wire radius used were $a=0.24 \mathrm{~cm}$ and $a=0.20 \mathrm{~cm}$.

\section{Computational Results and Interpretation}

The antenna designated LPDA-1 exhibited only one anomaly of moderate strength, at $735 \mathrm{MHz}$, so LPDA-2 was designed to produce two stronger anomalies. Antenna LPDA1 was studied extensively but LPDA-2 produced results better suited to presentation, so LPDA-1 will not be discussed further.

The backlobe level, with respect to the front lobe, of LPDA2 is shown in Fig. 2. This antenna has two anomalous back lobe peaks at $752 \mathrm{MHz}$ and $1060 \mathrm{MHz}$, respectively, the second peak being located just outside of the operating frequency band of $500-1000 \mathrm{MHz}$. The current distributions at the anomalous back lobe peak frequencies are unusual, compared to the normal current distributions at frequencies outside of the narrow resonant frequency bands, as illustrated in Fig. 3.

In Fig. 3, the normalized amplitude and the phase of the current distribution along the feed line are shown, and the normalized amplitude of the base current of each dipole element is also plotted. This figure shows that at the anomalous frequency of $752 \mathrm{MHz}$ those dipole elements behind the active region (in this case the three longest dipole elements) are excited approximately in proportion to the feed line current, but at the normal frequency of $835 \mathrm{MHz}$ these dipole elements remain unexcited and only the normal

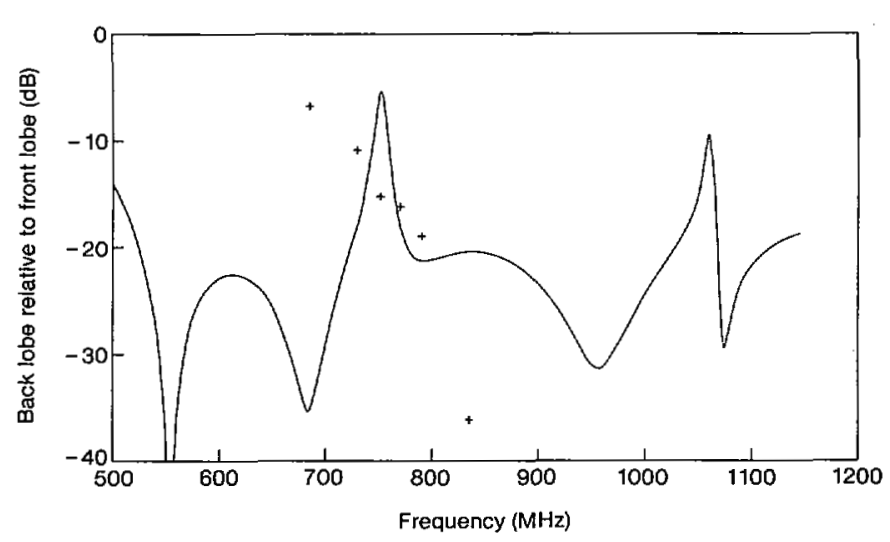

Fig. 2. Back lobe level with respect to front lobe for LPDA-2. The computed points $(+)$ are the consequence of deleting the far fields of the three longest dipoles from the computation.

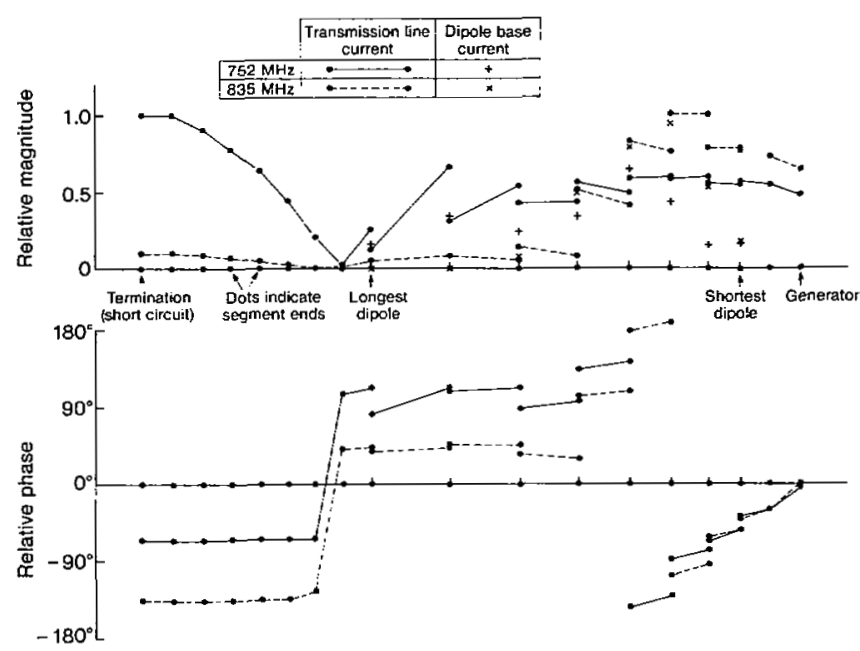

Fig. 3. Comparison of current distributions under normal conditions ( 835 $\mathrm{MHz}$ ) and anomalous conditions (752 MHz), for LPDA-2.

radiating region exists. It appears that at the anomalous frequency these excited longest dipole elements form another radiating region different from the main radiating region, so it may be called the anomalous radiating region. The peak of the anomalous radiating region is located at a point $\lambda / 2$ in front of the termination, where $\lambda$ is the free space wavelength. Characterized by the high feed line current at the termination and the variation of current magnitude and phase, the feed line resonance is clearly visible, and also it can be seen from the dipole base currents that the anomalous radiating region is excited by this feed line resonance. Similar conclusions may be drawn from the analysis of the second anomalous peak (1060 MHz, not shown) and in this case the location of the peak of the anomalous radiating region is located a distance $\lambda$ in front of the termination.

The computation of separately summing the radiation far fields of the dipole elements in the main radiating region and in the anomalous radiating region was performed. In Fig. 2, the back lobe levels of the radiation far field of the five shortest dipole elements involved in the main radiating region are marked at several frequencies around the resonant frequency band, so it is not difficult to conclude that the resonant back lobe far field is mainly generated by the longest dipole 
elements involved in the anomalous radiating region. With this understanding of the source of the anomalous behavior, the explanations of some known remedy methods for the anomalies are obvious. For example, consider attaching a resistor at the termination (even if it is not perfectly matched) or shorting the feed line immediately behind the longest element: in the former situation the transmission line resonance does not occur because of the damping imposed on the multiple reflections between the termination and the stop region near the $\lambda / 2$ dipole; in the latter situation no dipole element may be excited by the transmission line resonance if the boom length is shorter than $\lambda / 2$. These two known remedy methods [1] were confirmed in the computer analysis.

A few words should be given to [1], in which anomalous behavior was described and was thoroughly investigated, especially with regard to the relationship between anomalous performance and the value of the parameter $\tau$. However, some conclusions about the source of the anomalies were not so precise as in the present work. Although the splitting phenomenon of the radiating region was noted in [1], the formation of a completely new radiating region was not recognized, nor (of course) was its location identified.

\section{Effect of Thickness of Dipole Elements}

In most practical situations, the feed line of an LPD antenna is long enough to permit at least one resonance. Furthermore, attaching a matched resistor may be difficult for mechanical, electrical or economic reasons. Without a resistive termination, if a small part of the generator energy passes through the radiating region, the transmission line resonance must be excited and the anomalies must occur. Under these circumstances, the only effective remedy for the anomalies is to improve the coupling between dipole elements and feed line over a broad operating frequency band, to reduce the excited level of the transmission line resonance.

Because the variation with frequency of the input impedance of a thick cylindrical dipole is relatively smooth compared to that of a thin cylindrical dipole, using thick dipole elements should improve the coupling between dipole element and feed line and reduce the anomalous back lobe level. This attempt was made in the computer analysis. Fig. 4 shows the variations of the anomalous back lobe level of LPDA-2 with increasing dipole radius while the characteristic impedance of the feed line $Z_{0}$ remains constant. The result of a $4 \mathrm{~dB}$ reduction of the anomalous back lobe level caused by a 75 percent increase in dipole radius suggested that further increases in effective dipole radius should be tried.

\section{InPut Impedance Characteristics of Wide-Angle}

Triangular-Outline Dipoles with Central Wires

Wide-angle solid biconical dipoles have attractive broadband input impedance characteristics [4], but in practice it is difficult to employ them in LPD antennas. Wire simulations of the biconical dipoles are mechanically realistic, but the single planar triangular-outline dipoles do not quite have the desired broad-band impedance characteristics [4], [5]. An effort was made to improve the input impedance characteristics of a single planar triangular-outline dipole by simply adding a

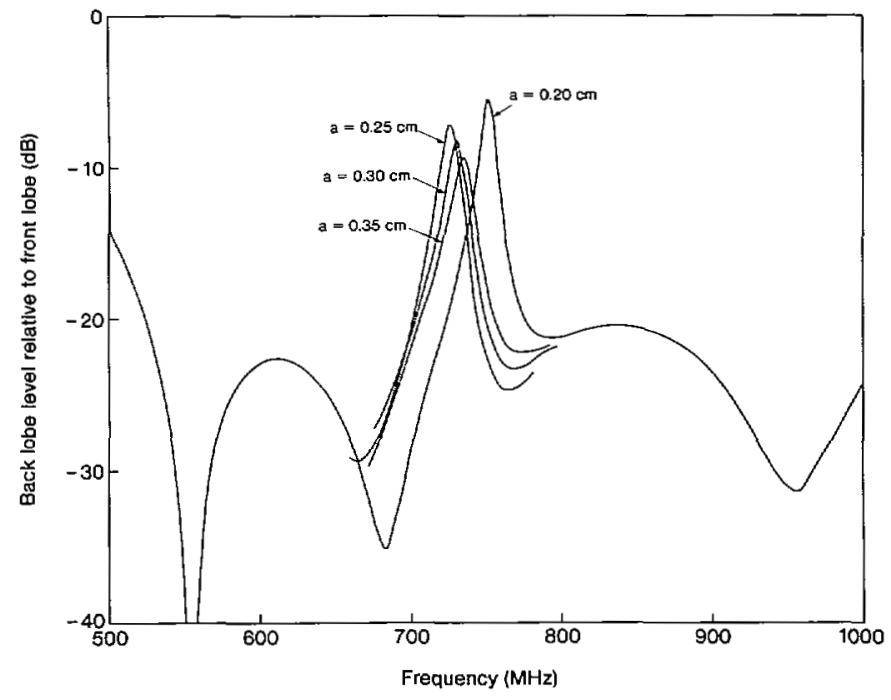

Fig. 4. Variations of the anomalous back Jobe level, with respect to the front lobe, with increasing radius " $a$ " of dipole elements and feed line while $Z_{0}$ of feed line remains constant: LPDA-2.

central wire to each monopole. By this means, a noticeable improvement in the input impedance characteristic is obtained, as well as an additional benefit of mechanical firmness. A comparison of the input impedance characteristics of the cylindrical dipole, the triangular-outline dipole and the triangular-outline dipole with central wires is given in Fig. 5 .

\section{Computer Analysis of Log-Periodic}

Triangular-Outline-Dipole Antennas with Central Wires

A log-periodic dipole antenna using the triangular-outline dipoles with central wires, named LPTDA-1, was designed with $\tau$ and the distances between corresponding dipole elements the same as in LPDA-2. Because the resonant electrical half-length of the wide-angle triangular-outline dipole with central wires shifts to a small value, to about $53^{\circ}$ from about $81^{\circ}$ for a straight wire (see Fig. 5), so the halflength of the corresponding dipole element in LPTDA-1 was reduced by $1 / 3$. LPTDA-1 has the following parameters: $\tau=$ $0.86, \sigma=0.124, \alpha=15.8^{\circ}, a=0.20 \mathrm{~cm}, Z_{0}=104 \mathrm{ohms}$, $s$ adjustable from $16 \mathrm{~cm}$ to $0.3 \mathrm{~cm}$, and the flare angle $\theta$ of all the triangular-outline dipoles is $60^{\circ}$. It is worth noting that the boom length between the longest and shortest elements of the LPD antenna having the same log-periodic parameters $\tau$ and $\sigma$, named LPDA-3, is greater by 50 percent compared to the boom length of LPTDA-1.

A similar numerical technique was employed with LPTDA1, each triangular-outline monopole being divided into seven segments with each side wire two segments and each central wire one segment, so the whole antenna was divided into 146 segments. More precisely, the computer analysis with each central wire divided into two segments and the whole antenna 162 segments was also carried out at several frequencies, the results showing that the error caused by treating each central wire as one segment is almost negligible, so the computer analysis of LPTDA-1 was performed in detail with 146 segments.

The back lobe level, with respect to the front lobe, of 


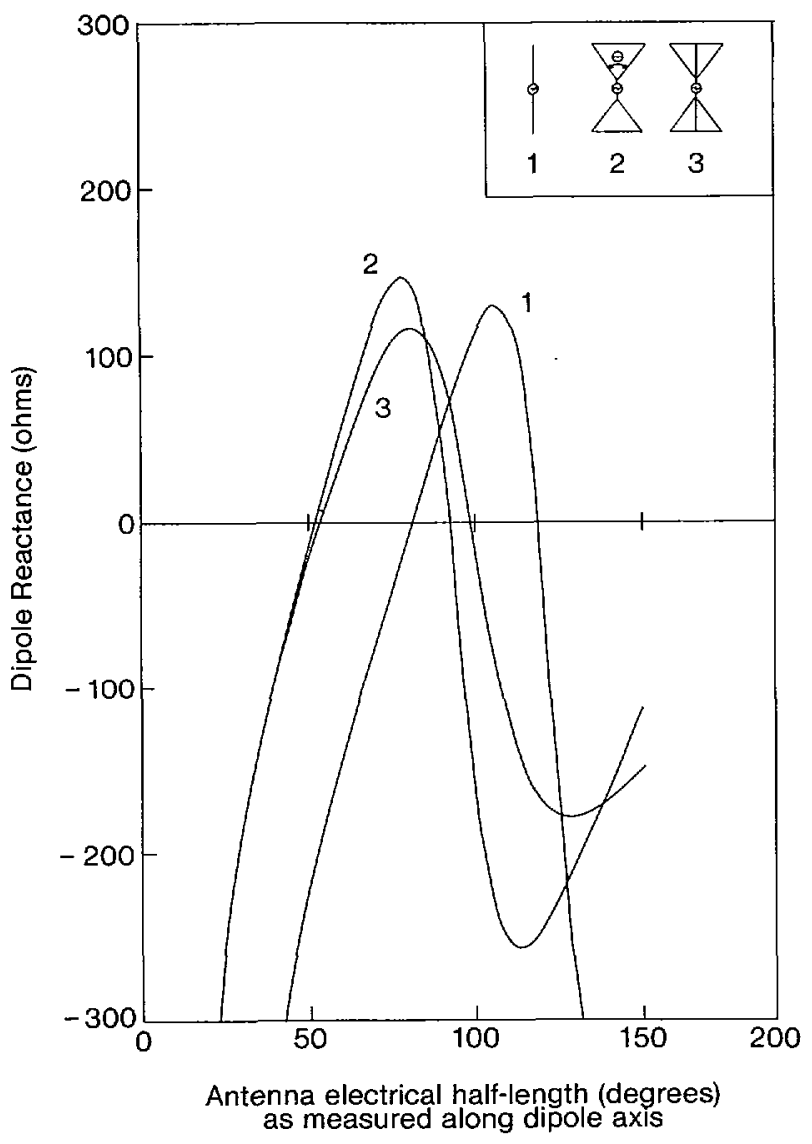

(a)

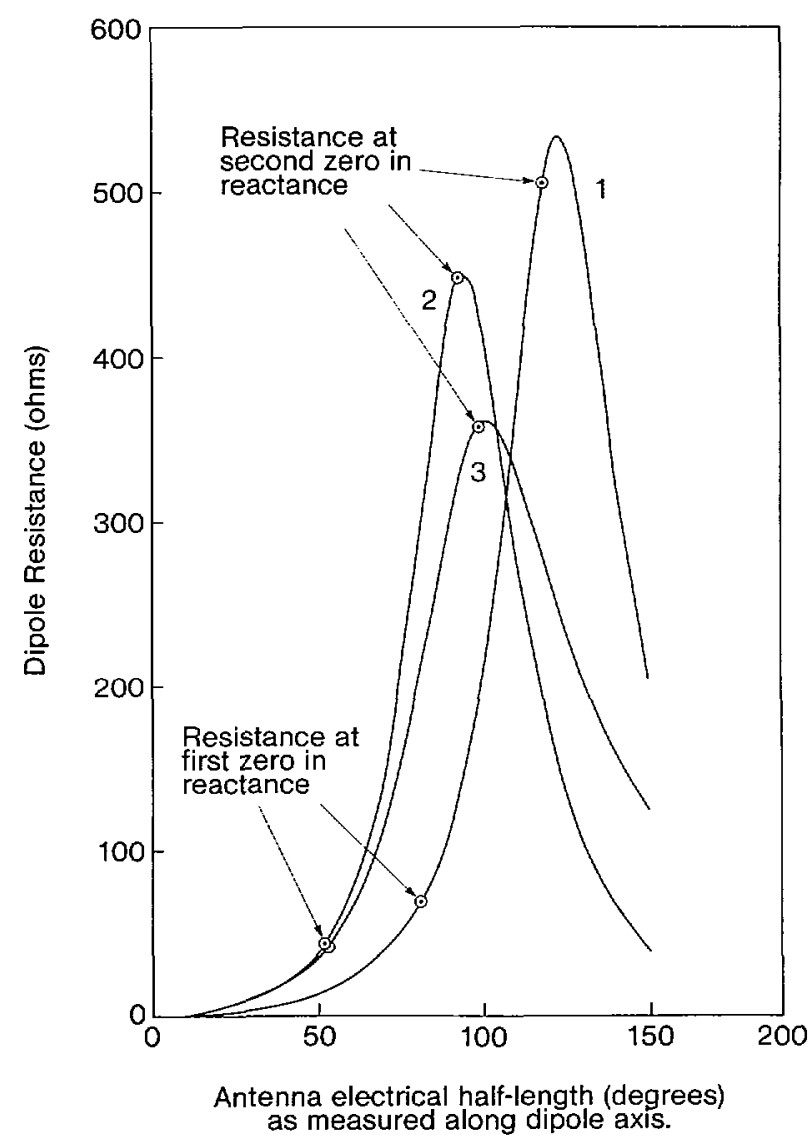

(b)

Fig. 5. Comparison of input impedance characteristics of a cylindrical dipole, a triangular-outline dipole and a triangular-outline dipole with central wires: $a=0.24 \mathrm{~cm}, \theta=60^{\circ}$. (a) Reactance. (b) Resistance.

LPTDA-1 is shown in Fig. 6, in which an almost invisible anomalous peak appears at $790 \mathrm{MHz}$, its level being $22 \mathrm{~dB}$ below the front lobe. For comparison, the back lobe levels of the corresponding log-periodic triangular-outline dipole antenna without central wires, named LPTDA-2, and of LPDA3 , which has the same parameters $\tau, \sigma, \alpha, a$ and $Z_{0}$ as LPTDA1 , are also displayed in Fig. 6. For LPTDA-2, a visible anomalous peak appears at $753 \mathrm{MHz}$ with its level being 17.4 $\mathrm{dB}$ below the front lobe. Note that the anomalous peak of LPTDA-2 is moderately flat compared to those peaks of LPDA-2 and LPDA-3, and the anomalous peak of LPTDA-1 is so flat that it is almost invisible. This feature shows that the log-periodic antenna with wide-angle triangular-outline dipoles with central wires has the desired freedom from anomalously high back lobes, at least in theory and for one set of parameters.

\section{EXPERIMENTAL StUdy of Log-Pertodic}

Triangular-Outline-Dipole Antennas with Central Wires

\section{Antenna Structure and Experimental Method}

Two experimental log-periodic triangular-outline-dipole antennas with central wires were built. For comparison with the computer analysis, these antennas were designed to be the same as the model analyzed by computer, that is, all the dipole elements and the feed line were made of wires of the same

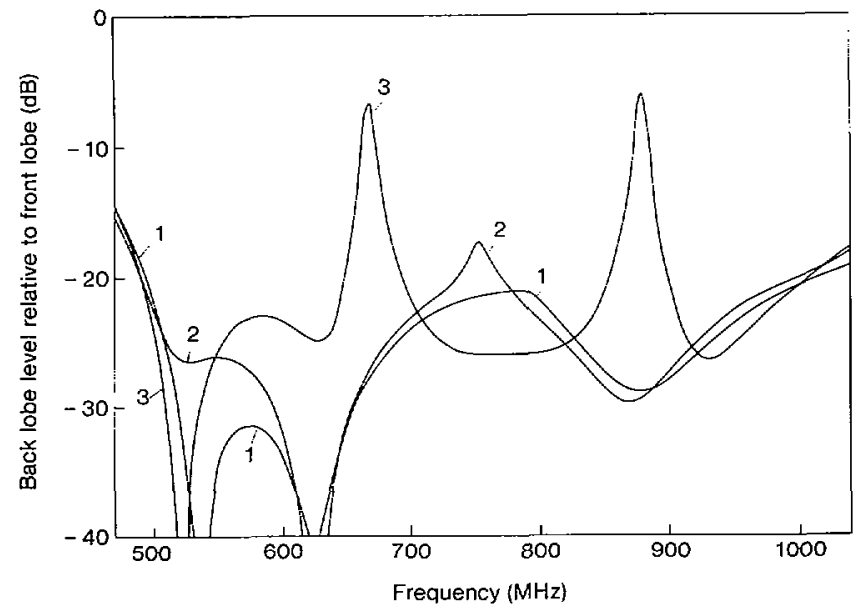

Fig. 6. Comparison of back lobe levels: 1: LPTDA-1; 2: LPTDA-2; 3: LPDA-3.

diameter, but for ease of construction all the dipole elements are made of wires of square cross-section of equivalent radius. The two antennas are identical for the purpose of making absolute gain measurements. They have the same parameters as those of LPTDA-1 except $a=0.24 \mathrm{~cm}$ because wire of $0.20 \mathrm{~cm}$ radius is not commercially available. Each antenna has a movable shorted termination (a movable end clamp). Fig. 7 shows a photograph of such an experimental antenna. 


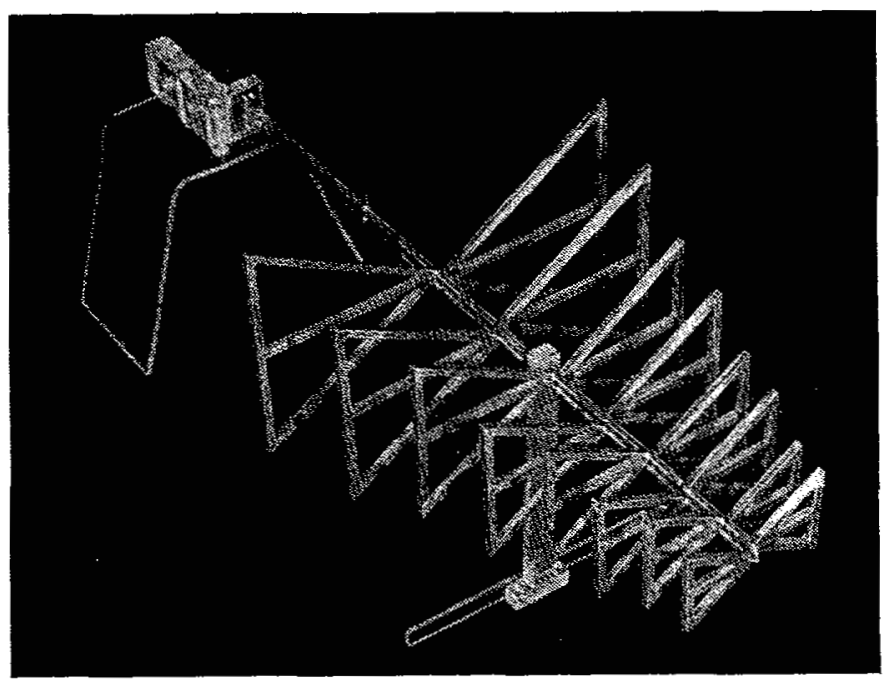

Fig. 7. An experimental log-periodic triangular-outline-dipole antenna with central wires.

The experimental study was carried out in an anechoic chamber, one antenna being used as the receiving antenna and the other as the transmitting antenna. The swept-frequency far-field measurement technique was used, in which the transmitting antenna was fixed and the receiving antenna rotated. The distance between the antennas was at least $2.4 \mathrm{~m}$ and the results were found to be independent of this distance. At first, the fundamental characteristics of the receiving antenna were measured under conditions of the computer analysis, that is, setting the length of the feed line section from the termination to the longest dipole at the value $s=15 \mathrm{~cm}$ and setting $Z_{0}=104 \mathrm{ohms}$. Then the characteristics were measured with the short circuit close to the largest element $(s$ $=0.3 \mathrm{~cm})$ and $/$ or with a lower characteristic impedance $\left(Z_{0}\right.$ $=80 \mathrm{ohms}$ ). Because the measured results showed no difference when changing the length $s$ on the transmitting antenna, the length $s$ on the transmitting antenna was either fixed or was set equal to the same length as in the receiving antenna, as needed.

\section{Performance With the Same Parameters as in the Computer Analysis}

Radiation Patterns: The measured swept-frequency farfield $H$-plane patterns are shown in Fig. 8, which demonstrates satisfactory performance over the operating frequency band, from 500 to $1000 \mathrm{MHz}$. The back lobe peak at $710 \mathrm{MHz}$ in Fig. 8 has been identified as an anomalous peak because its frequency is changed by moving the end clamp. This peak is at a level of $-19 \mathrm{~dB}$ relative to the front lobe. Asymmetry resonance phenomena [6] were also noted in the corresponding $E$-plane pattern (not shown), but the resulting side radiation was always at a level lower than $-22 \mathrm{~dB}$ relative to the front lobe: this shows that a satisfactory degree of symmetry was achieved in spite of the complexity of the antenna geometry.

Directivity and Gain: From measured $H$-plane and $E$ plane patterns, the half-power beamwidths in those planes were determined. By the use of Kraus' approximate formula

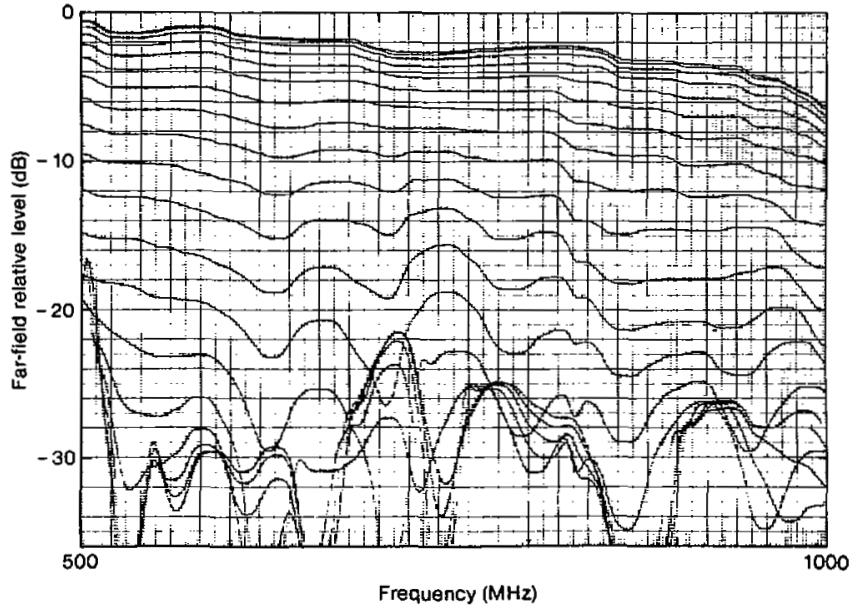

Fig. 8. Swept-frequency far-field patterns: $s=15 \mathrm{~cm}, Z_{0}=104 \mathrm{ohms}$, left half $H$-plane with $10^{\circ}$ rotation increments (the top trace is in the boresight direction, and each successive trace below is for an additional $10^{\circ}$ rotation of the antenna away from the boresight direction, terminating at $180^{\circ}$ from boresight where there are back lobe peaks just above $500 \mathrm{MHz}$ and just above $700 \mathrm{MHz}$ ). Frequency scale is linear.

[7]

$$
D_{0}=10 \log _{10} \frac{41253}{\theta_{E} \phi_{H}}
$$

the directivity $D_{0}$ of the antenna under test was obtained (this formula is known to produce overestimates in the vicinity of one $\mathrm{dB}$ ). For two identical antennas, when they are polarization-matched and aligned for maximum directional radiation, the maximum power gain $G_{0}$ is given by [8]

$$
G_{0}=\frac{1}{2}\left[20 \log _{10} \frac{4 \pi R}{\lambda}+10 \log _{10} \frac{P_{r}}{P_{t}}\right]
$$

where $P_{r}$ is the received power and $P_{t}$ is the transmitted power. The ratio of $P_{r} / P_{t}$ in $\mathrm{dB}$ can be determined by the swept-frequency far-field measurements, hence the gain $G_{0}$ obtained.

In the case of $s=15 \mathrm{~cm}, Z_{0}=104$ ohms and antenna separation of $2.4 \mathrm{~m}$, the measured $D_{0}$ and $G_{0}$ are listed in Table I, and for comparison the computed gain is also given. A fairly good agreement between the computed and the experimental results is demonstrated.

Input Impedance: The input impedance, which was measured by using a HP 8505A network analyzer, is given in Fig. 9. It shows no anomalous behavior over the full operating frequency band of $500-1000 \mathrm{MHz}$. The impedance values lie within a circle of voltage standing-wave ratio (VSWR) $=2.0$ with respect to a $60 \mathrm{ohm}$ average over the full band, and the maximum VSWR drops to 1.5 over the range $500-800 \mathrm{MHz}$.

\section{Performance With Short Circuit Close To Largest Element}

As the shorted termination was moved closer to the largest element, the anomalous back lobe peak at $710 \mathrm{MHz}$ moved to higher frequencies, ultimately higher than $1000 \mathrm{MHz}$ for $s<$ $1 \mathrm{~cm}$ as seen from the $H$-plane pattern shown in Fig. 10. As 
TABLE I

DIRECTIVITY AND GAIN (WITH RESPECT TO AN IDEAL ISOTROPIC RADIATOR)

\begin{tabular}{l|cccccc}
\hline \multicolumn{1}{|c|}{ Frequency (MHz) } & 500 & 600 & 700 & 800 & 900 & 1000 \\
\hline $\begin{array}{l}\text { Measured } \\
\text { Directivity (dB) }\end{array}$ & 7.7 & 8.0 & 7.8 & 8.0 & 8.4 & 7.9 \\
$\begin{array}{l}\text { Measured } \\
\text { Power Gain (dB) }\end{array}$ & 6.3 & 6.9 & 6.7 & 7.2 & 7.0 & 6.3 \\
$\begin{array}{l}\text { Computed } \\
\text { Power Gain (dB) }\end{array}$ & 6.9 & 7.4 & 7.2 & 7.4 & 7.5 & 7.0 \\
\hline
\end{tabular}

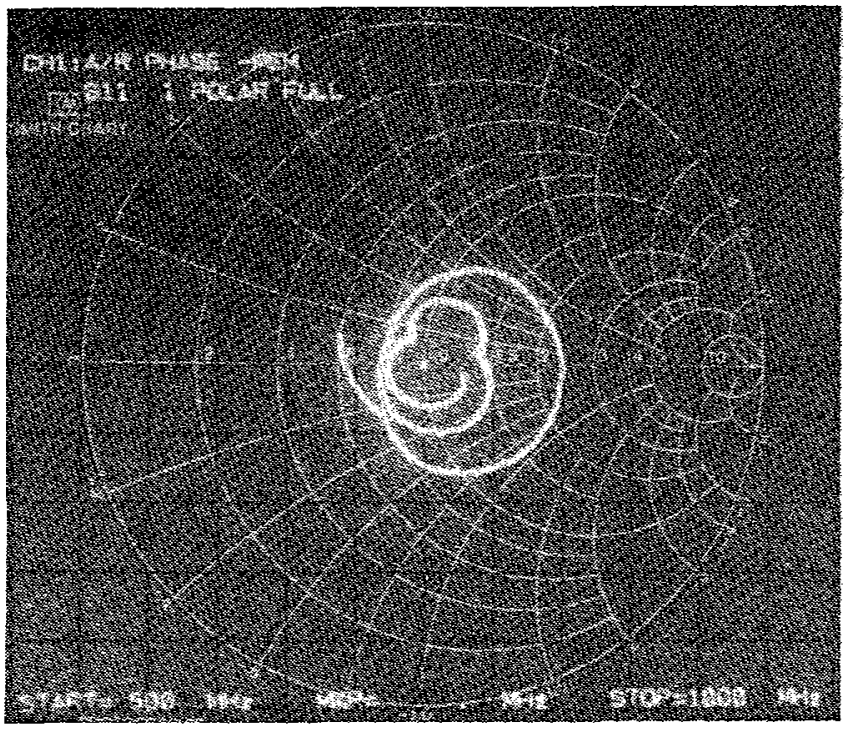

Fig. 9. Input impedance: $s=15 \mathrm{~cm}, Z_{0}=104 \mathrm{ohms}$.

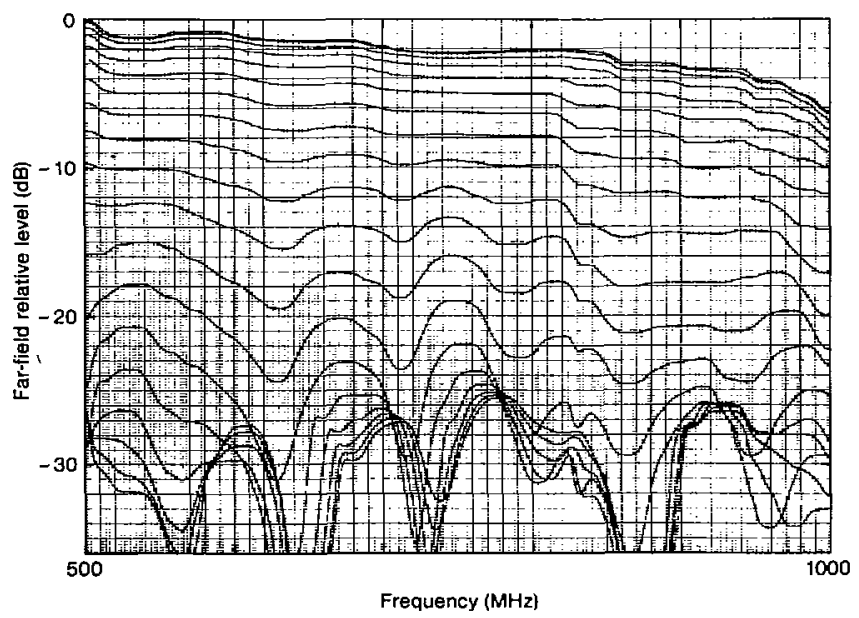

Fig. 10. Swept-frequency far-field $H$-plane pattern: $s=0.3 \mathrm{~cm} ; Z_{0}=104$ ohms; left half $H$-plane with $10^{\circ}$ rotation increments (see explanation in caption to Fig. 8).

the shorted termination was moved, the back lobe level passed through two maxima relative to the front lobe, $-19 \mathrm{~dB}$ at 710 $\mathrm{MHz}$ for $s=15 \mathrm{~cm}$, and $-17.5 \mathrm{~dB}$ at $970 \mathrm{MHz}$ for $s=3 \mathrm{~cm}$. For values of $s$ in the range 6 to $11 \mathrm{~cm}$, the back lobe was lower than $-20 \mathrm{~dB}$. Note that the high back lobe level at 500 $\mathrm{MHz}$ in Fig. 8 drops by $10 \mathrm{~dB}$ in Fig. 10, suggesting that a short circuit at the longest dipole is the optimum reactive feeder termination because it increases the operating bandwidth at both low and high frequencies.

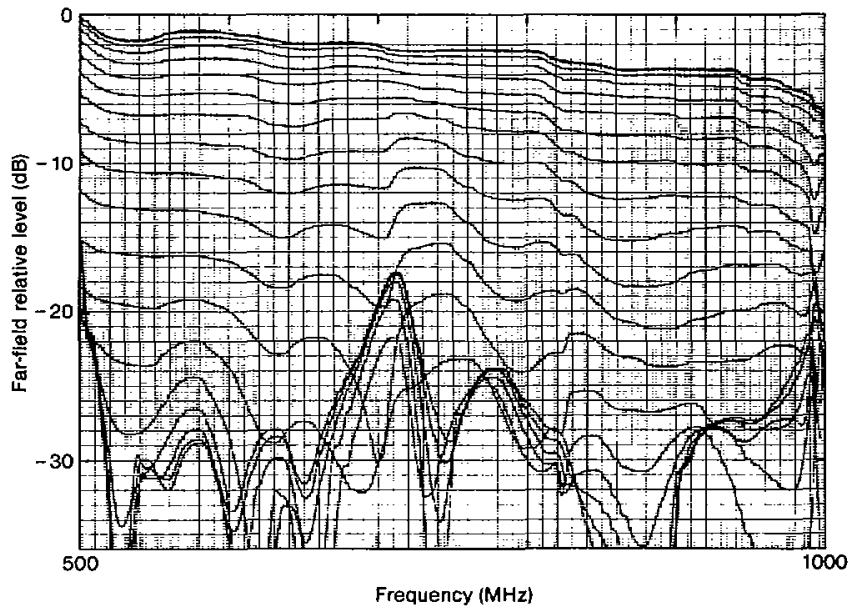

(a)

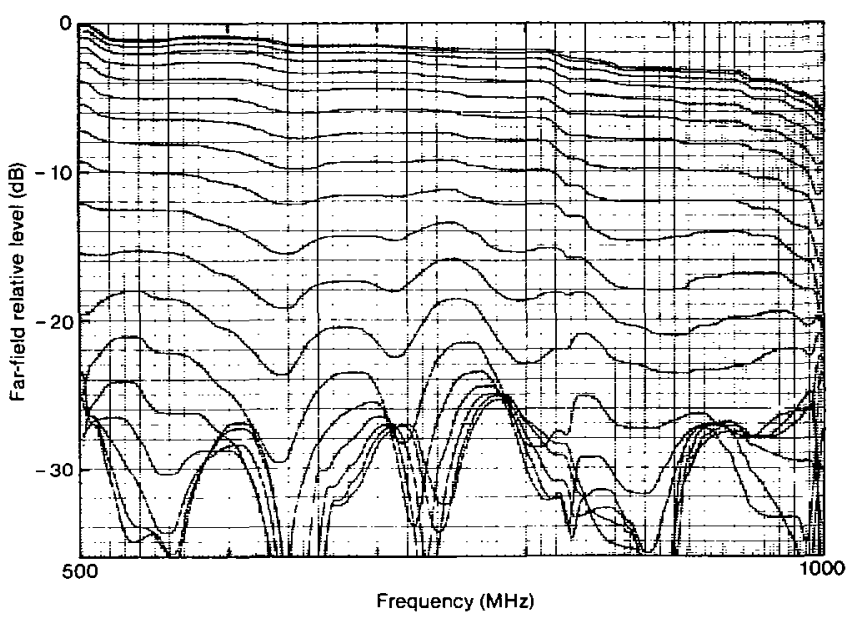

(b)

Fig. 11. Swept-frequency far-field $H$-plane pattern: left half $H$-plane with $10^{\circ}$ rotation increments; $Z_{0}=80$ ohms. (a) $s=15 \mathrm{~cm}$. (b) $s=0.3 \mathrm{~cm}$ (see explanation in caption to Fig. 8).

\section{Performance With Low Feedline Characteristic Impedance}

In order to observe the performance of the antenna with parameters likely to worsen the anomalies, the characteristic impedance $Z_{0}$ of the receiving antenna was reduced to 80 ohms. When $s=15 \mathrm{~cm}$ the anomalous back lobe peak, which is shown in Fig. 11(a), rose to a level $4 \mathrm{~dB}$ higher than in the case with $Z_{0}=104$ ohms shown in Fig. 8. But when the end clamp was placed close to the largest element, $s=0.3 \mathrm{~cm}$, the $H$-plane pattern presented in Fig. 11(b) turned out to be almost identical to Fig. 10, except for the fact that the anomalous resonance had moved to a frequency slightly lower than 1000 $\mathrm{MHz}$. The average impedance had moved to $50 \mathrm{ohms,}$ with VSWR performance similar to that at $Z_{0}=104 \mathrm{ohms}$ and also independent of end-clamp position.

\section{ConCLUSION}

The anomalous behavior of LPD antennas has been analyzed by computer. The source of the anomalous back lobe radiation is the anomalous radiating region, which is excited by the transmission-line resonance of the feedline shorted at the termination. 
The wide-angle triangular-outline dipoles with central wires exhibit the desired broad-band input impedance characteristics for use in LPD antennas. A log-periodic dipole antenna using the wide-angle triangular-outline dipoles with central wires has been designed, and the computer analysis of this antenna shows that the anomalous high backlobe level over a narrow frequency band is depressed to a negligible level.

Experimental log-periodic triangular-outline-dipole antennas with central wires have been built and tested. The results of the experimental study show good agreement with the computer analysis. This new construction method produces an LPD antenna which is largely free of anomalous resonance effects, in spite of having a relatively low value of scale factor $(\tau=0.86)$, and in spite of being well matched to cables in the $50-60 \mathrm{ohm}$ range.

\section{ACKNOWLEDGMENT}

For the duration of the research, $\mathrm{Z}$. Gong held a research fellowship provided by the government of the People's Republic of China.

\section{REFERENCES}

[1] C. C. Bantin and K. G. Balmain, "Study of compressed log-periodic dipole antennas,"' IEEE Trans. Antennas Propagat., vol. AP-18, pp. 195-203, Mar. 1970.

[2] K. G. Balmain, C. C. Bantin, C. R. Oakes, and L. David, "Optimization of log-periodic dipole antennas," IEEE Trans. Antennas Propagat., vol. AP-19, pp. 286-287, Mar. 1971.
[3] J. H. Richmond, "Computer program for thin-wire structiures in a homogeneous conductive medium," NASA CR-2399, June 1974.

[4] G. H. Brown and O. M. Woodward, "Experimentally determined radiation characteristics of conical and triangular antennas," $R C A$ Rev., vol. 13 , pp. $425-452$, Dec. 1952.

[5] C. E. Smith, C. M. Butler, and K. R. Umashankar, "Characteristics of a wire biconical antenna," Microwave J., vol. 22, pp. 37-40, Sept. 1979.

[6] K. G. Balmain and J. N. Nkeng, "Asymmetry phenomenon of logperiodic dipole antennas," IEEE Trans. Antennas Propagat., vol. AP-24, pp. 402-410, July 1976.

[7] J. D. Kraus, Antennas. New York: McGraw-Hill, 1950, p. 25.

[8] IEEE Standard Test Procedures for Antennas, IEEE Standard 1491979 , p. 96

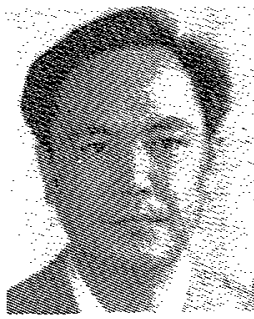

Zhong-lin Gong was born in Chungking, Szechuan Province, China, on May 10, 1940. He graduated from the Department of Radio-Electronics of Peking University, China, with a degree in electron physics.

After graduation, he was with the Department of Radio-Electronics, Peking University. From 1981 to 1983 he was at the Department of Electrical Engineering, University of Toronto, Canada, as a Visiting Scholar with the China-Canada Scholarly Exchange Program. He returned to Peking University in 1983 where he is now an Associate Professor. His current research interests include transient electromagnetic fields, satellite communication antennas, and biological effects of electromagnetic fields.

Keith G. Balmain (S'56-M'63-SM'85), for a photograph and biography please see page 260 of the March 1983 issue of this TRANSACTIONS.

\title{
A Network Model of a Feedthrough Phased Array Lens of Printed Dipole Elements
}

\author{
RUEY-SHI CHU, MEMBER, IEEE, KUAN-MIN LEE, MEMBER, IEEE, AND NAM SAN WONG, MEMBER, IEEE
}

\begin{abstract}
An easy-to-use simplified network representation for the internal multiple reflection model of feedthrough phased array lens antenna elements is presented. This model makes use of the readily known active radiation impedances of an infinite array rather than the self and mutual impedances between array elements. In particular, this model is applied to a printed dipole lens array case, for which the computation of the radiation impedance is described. Numerical results for this lens array are given to show the aperture distribution variations due to the lens internal multiple reflection, and the gain falloff as a function of scan angle.
\end{abstract}

\section{INTRODUCTION}

$I^{\prime}$ A SPACE-FED feedthrough phased array lens antenna system, multiple reflections will occur in each lens element

Manuscript received January 26, 1986; revised May 29, 1986.

The authors are with the Antenna Department, Ground Systems Group, Hughes Aircraft Company, Box 3310, Fullerton, CA 92634

IEEE Log Number 8610054. due to the mismatches of the radiation impedances of the radiating elements on the pick-up side and the reradiation side, the feed lines, and the phase shifters. These multiple reflections in each lens element will produce ripples in the amplitude and phase distributions across the lens aperture. Thus the antenna gain and sidelobe levels will be degraded. An analysis of phased array lenses has been given by Schwartzman and Topper [1]. There, an equivalent network representation of a feedthrough lens array, which takes into account of mutual coupling between the array elements, was given. A set of coupling equations, which involve the self- and mutual impedances between the array elements, were obtained. By using the self- and mutual impedances derived by Carter [2] for the array of thin-wire dipoles, they presented numerical results for a linear lens array of 51 element pairs of thin-wire halfwavelength dipoles to illustrate the effect of the internal multiple reflection on the lens aperture distribution. Unfortunately, the self- and mutual impedances of an array consisting 